\title{
Directional Dark Matter Search with the Fine Grained Nuclear Emulsion
}

\author{
T. Asada*, T. Katsuragawa, M. Yoshimoto, K. Hakamata, M. Ishikawa, A. Umemoto, \\ S. Furuya, S. Machii, K. Kuwabara, T. Nakano \\ Graduated School of Science, Nagoya University, Furo-cho, Chikusa-ku, Nagoya, Japan \\ E-mail: asadaeflab.phys.nagoya-u.ac.jp
}

\section{T. Naka}

Institute of Advanced Research, Nagoya University, Furo-cho, Chikusa-ku, Nagoya, Japan

Kobayashi-Maskawa Institute for The Origin of Particles and The Universe, Nagoya University,

Furo-cho, Chikusa-ku, Nagoya, Japan

\section{Y. Suzuki}

Japan Synchrotron Radiation Research Institute/Spring-8, Mikazuki, Hyogo, Japan

\section{Nakamura, O. Sato}

EcoTopia Science Institute, Nagoya University, Furo-cho, Chikusa-ku, Nagoya, Japan

\begin{abstract}
The dark matter is one of the most serious problems of physics. Directional dark matter search plays important role to prove their existence from new aspect. We are challenging it with detecting sub-micron track in fine grained nuclear emulsion. The emulsion can provide both directional search and large mass target.We improved emulsion quality and new readout technique which provides high sensitivity and low background for sub-micron tracks.
\end{abstract}

KMI International Symposium 2013 on "Quest for the Origin of Particles and the Universe" 11-13 December, 2013

Nagoya University, Japan

\footnotetext{
*Speaker.
} 


\section{1. introduction}

From 1930, many observations have indicated the existence of the dark matter. The result of Plank 2013 showed that dark matter is more than 5 times of the ordinary matter. Rotation velocity curve of the galactic system means the existence of dark matter in local scale.

There are many experiments for direct detection of Weakly Interacting Massive Particles. WIMP is a candidate of dark matter, and expected to recoil normal nucleus with very small cross section. Recently, some experiment said that they found WIMP signals, and their signals modulated in sync with the revolution of the earth[1][2] [3]. However some results of other experiments have denied their signal region[4], and the truth has not been clear yet.

\section{Directional search}

WIMP is considered to be trapped in the galaxy and the expectation value of WIMP in local region of solar system is $0.3 \mathrm{GeV} / \mathrm{cm}^{3}$. The direct dark matter search experiments detect the signal of recoiled atom which comes from the interaction with WIMP. They usually detect energy spectrum and distribution of event counts, and they can find WIMP wind modulation which comes from earth revolution. However there are some backgrounds which have suspicion of modulation depends on environment. On the other hand, the "directional" dark matter search can find the signals free from environmental modulation.

WIMP is considered to have lower velocity distribution than escape velocity of the galaxy and the solar system are moving towards Cygnus at $230 \mathrm{~km} / \mathrm{sec}$. These give large deviation to their distribution, which is like a wind. Directional dark matter searches find the direction of atom recoiled by deviated WIMP, so the distribution of direction should be also deviated. This is only depends on earth attitude and independent from ground environment, thus they can give strong proof for WIMP existence. (Fig 1)

We plan a direct dark matter search experiment with nuclear emulsion [5]. The emulsion have important abilities of directional search and large mass detection at the same time. Existing directional experiments use gaseous detector, so their target mass are limited up to gram scale. The emulsion has many experiences of large mass experiments up to ton scale. Simulation shows that we can reach DAMA region with less than $100 \mathrm{~kg}$ scale in principle. (Fig 3) The fault of emulsion is expected length of tracks. Their ranges are estimated to be less than micron order (Fig, 2). However we developed the method to detect them.

\section{Detector}

The emulsion is made by gelatin plate including small $\mathrm{AgBr}$ crystals. The crystal which particles passed generates large silver grain with developing treatment. These finally make lined silver grains where particle passed and we can tracking them. The quality of these crystals decides the theoretical detection limits. We succeeded to improve resolution and sensitivity of emulsion.

Micronization is very important because crystal size gives theoretical resolution and energy threshold. We succeed to make very small crystals than those of existing emulsions(Fig 5 ). These emulsions are called U-NIT, and the smallest one had $18 \mathrm{~nm}$ crystals in average. It means we can 


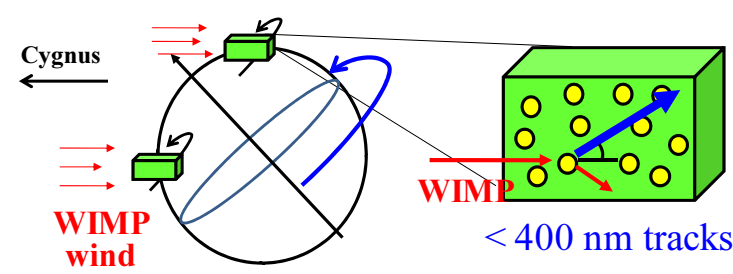

Figure 1: The Concept of directional dark matter search on earth. Direct rotation of emulsion detector allows the constant integration of signals from same direction in the space.

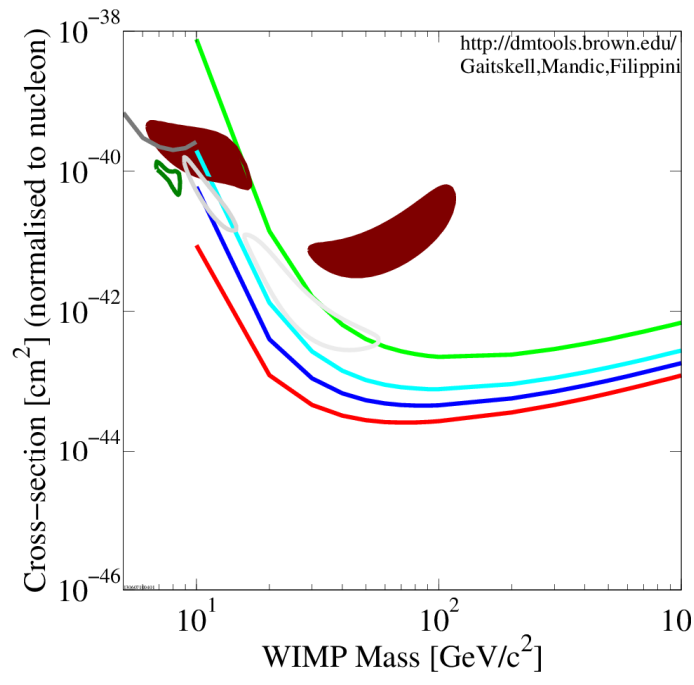

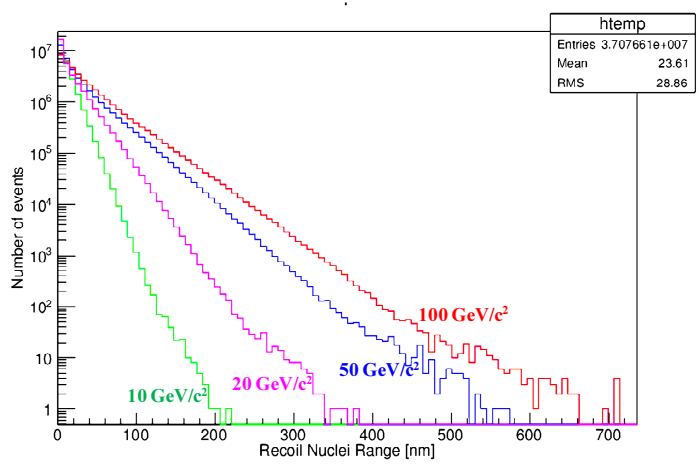

Figure 2: Expected recoil atom range with 10-100 $\mathrm{GeV}$ WIMP in $3.2 \mathrm{~g} / \mathrm{cm}^{3}$ emulsion.

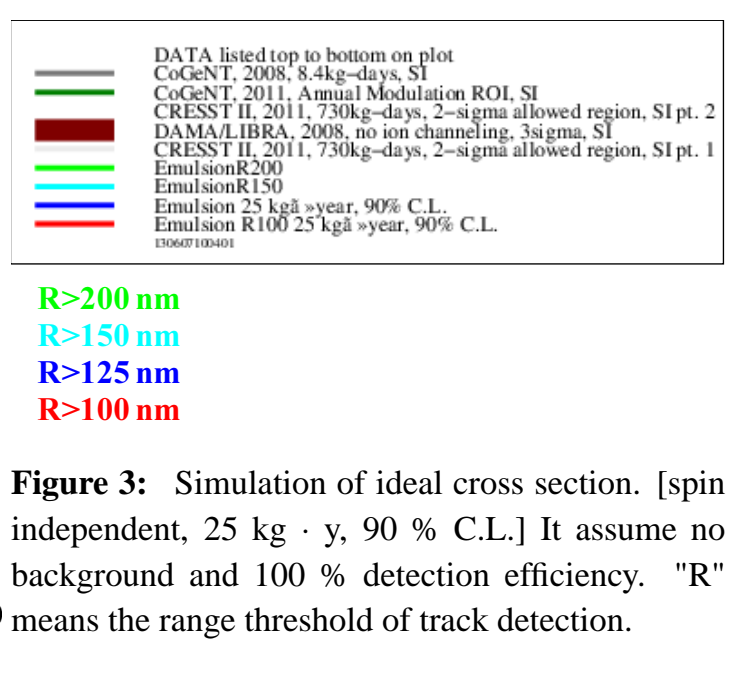

detect shorter than $100 \mathrm{~nm}$ tracks, in the other wards, around $35 \mathrm{keV}$ carbon track is detectable in theory.

We also studied crystal sensitivity with existing NIT type emulsion(Fig 4 ). Sensitivity decides detection efficiency of signal and background. There are some methods, and reducing electron-hole recombination inside crystal was most effective. This method gives around $90 \%$ response per 1 crystal for Carbon atom.

On the other hand, background $\gamma$-electron generates electrons. They are fewer than those of signals, but the amount of $\gamma$-electrons are large enough to cover signals. We reject them by introducing electron trap inside the crystal, and proved that their rejection is possible in theory.

\section{Readout system}

Signal candidates are selected with optical microscope, and checked with X-ray microscope. Optical analysis is important for large amount candidates, but their tracks are too short to distinguish each grain. We fit outline of clustered grains as ellipse, and succeed to detect around $200 \mathrm{~nm}$ tracks. Then passing candidates are confirmed with X-ray microscope by corresponding one-to- 


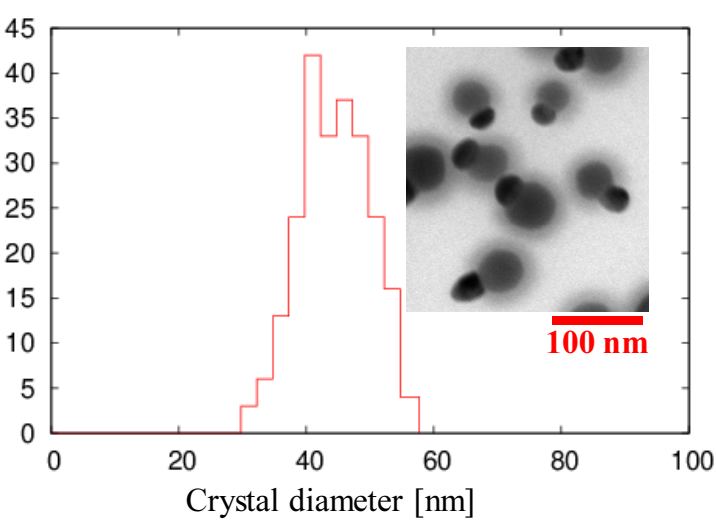

Figure 4: NIT type emulsion. Size of crystals and picture on TEM.

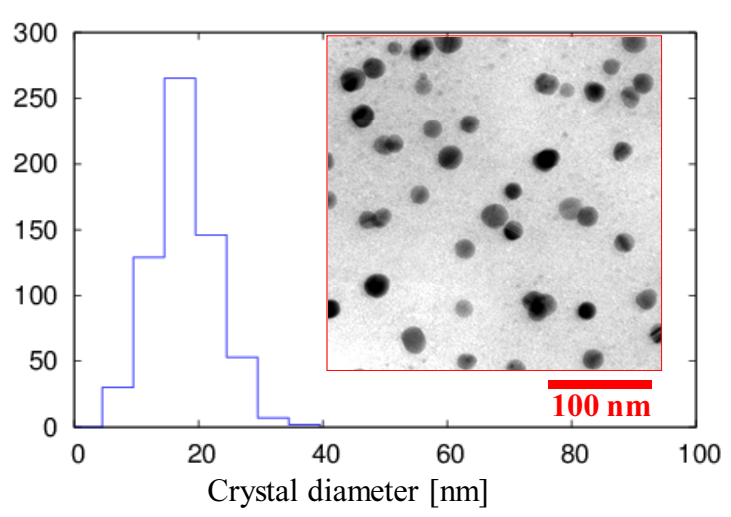

Figure 5: U-NIT type emulsion

\begin{tabular}{|c|c|c|}
\hline & NIT & U-NIT \\
\hline mean crystal size & $44.6 \pm 0.4 \mathrm{~nm}$ & $18.0 \pm 0.2 \mathrm{~nm}$ \\
\hline crystal density & $12 \mathrm{crystal} / \mu \mathrm{m}$ & $29 \mathrm{crystal} / \mu \mathrm{m}$ \\
\hline Crystal sensitivity for Carbon & $\sim 65 \%$ & $\sim 30 \%$ \\
\hline Sensitized crystal sensitivity for Carbon & $>90 \%$ & not yet \\
\hline Detectable range & $>200 \mathrm{~nm}$ for Carbon & $>100 \mathrm{~nm}$ for Carbon \\
\hline Tracking E threshold & $>80 \mathrm{keV}$ for Carbon & $>35 \mathrm{keV}$ for Carbon \\
\hline
\end{tabular}

Table 1: Feature of two type emulsion. Efficiencies are estimated with accelerated carbon ion.

one. X-ray microscope has $70 \mathrm{~nm}$ spatial resolution with non-destructive observing, and gives clear shape of each grain. This process was basically established, but still has weak points for amount of back ground contamination, and scanning speed of X-ray microscope.

We are studying new analysis with optical color information come from plasmon effect. Our silver grains fortunately have sensitive size for surface plasmon which strongly interacts to visible light(Fig 6). Observed spectrum would have the information of crystal size which concerns the energy deposit of passed particle. It will greatly help us to reject noise grains.

This effect works not only in a grain but also track. Signal track is constructed by closed crystals, and plasmon effect is quite sensitive in this gap distance. In addition to it, reflection light from track is polarized. Preliminary result shows that polarization direction is exactly match track direction(Fig 7). This technique would give us a new analysis method which provides both high speed and high resolution of extremely short tracks.

\section{Conclusion and Prospect}

The emulsion is good method to realize Directional dark matter search. We improved basic quality of detector to detect extreme short tracks, and proved new high resolution readout method which may allow us large mass experiment. Next step is the combination test of those techniques 

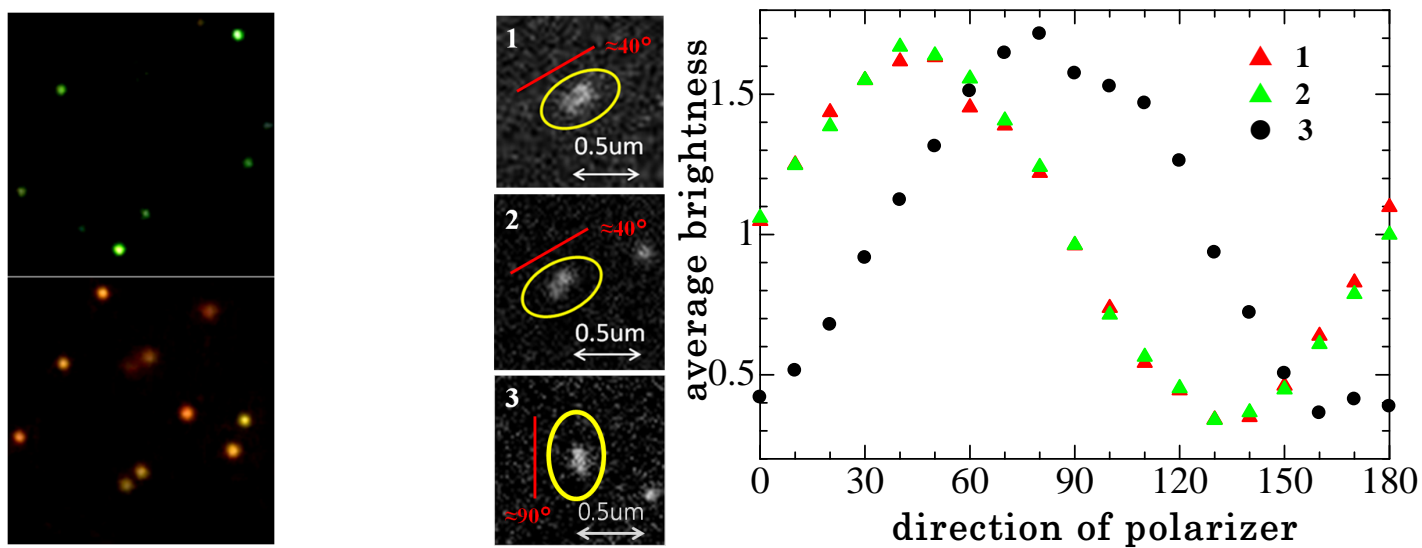

Figure 6: Left: Optical microscope images of small silver crystals under same lights. Top is $60 \mathrm{~nm}$ and bottom is $80 \mathrm{~nm}$ silver crystals.

Figure 7: Center : X-ray microscope image of closed silver crystals. Right : Brightness of reflected lights from closed silver particles of center pictures. Introduced light has $>760 \mathrm{~nm}$ wave length and reflected lights passed through polarizer.

and background run. We are planning small scale experiment for this in a year with these techniques.

\section{References}

[1] R.Bernabei et al, Eur. Phys. J.,C67, 39 (2010)

[2] G.Angloher et al., Eur. Phys. J C, 72, 1971 (2012)

[3] C.E. Aalseth et al., Phys. Rev. Lett. 107 (2011) 141301

[4] E. Aprile et al, Phys. Rev. Lett. 109, 181301 (2012)

[5] T. Naka et al., Nucl. Ins. Meth. A, 718 (2013) 519-521 